DOI: 10.1002/adem.((please add manuscript number))

\title{
Tunable, Flexible composite magnets for marine monitoring applications**
}

By Altynay Kaidarova, Mohammed Asadullah Khan, Selma Amara, Nathan Geraldi, Muhammad Akram Karimi, Atif Shamim, Rory.P. Wilson, Carlos.M. Duarte, and Jurgen Kosel *

[*] Prof. Jurgen Kosel, Altynay Kaidarova, Mohammed Asadullah Khan, Selma Amara Computer, Electrical and Mathematical Sciences \& Engineering (CEMSE)

4700 King Abdullah University of Science and Technology(KAUST)

Kingdom of Saudi Arabia, Thuwal 23955

Email: jurgen.kosel@kaust.edu.sa

Nathan Geraldi, Prof. Carlos.M. Duarte

Red Sea Research Center (RSRC)

4700 King Abdullah University of Science and Technology (KAUST)

Kingdom of Saudi Arabia, Thuwal 23955

Prof.Rory.P. Wilson

Swansea Lab for Animal Movement, Biosciences

Swansea University, Swansea SA2 8PP

Wales, United Kingdom (UK)

This paper presents flexible NdFeB-PDMS composite magnets with tunable magnetic and mechanical properties optimized for applications in corrosive marine environments. The magnetic and mechanical properties were studied for different NdFeB powder concentrations and the performance of the magnetic composites for different exposure times to sea water investigated systematically. The remanence and saturation magnetizations could be tailored by the powder concentration, whereby up to $70 \%$ wt concentration could be employed without compromising the integrity of the magnets. The elastic modulus of the composite magnets was about $10^{5}$ times lower than the one of a bulk permanent magnet. This ensures a high bending flexibility, which allows the magnets to be attached to curved surfaces as illustrated for a giant clam, crab and turtle. At the same time, the weight of the composite magnets is reduced by a factor of about 10, which poses less burden to animals' natural free movement. Without a protective layer, the composite magnets lose more than $50 \%$ of their magnetization after 51 days in seawater. However, the durability of the composite magnets has been improved considerably by using polymer coatings. Parylene $C$ was the most effective for this, providing 
corrosion resistance, flexibility and enhanced biocompatibility. Parylene C films of $2 \mu \mathrm{m}$ and $4 \mu m$ thicknesses provided excellent protection of the magnetic composite in corrosive aqueous environments for 65 and 82 days, respectively. By combining the composite magnets with tunnel magnetoresistance sensors, a magnetic animal monitoring system was established that was used to track the behavior of giant clam, crab and turtle.

\section{Introduction}

Understanding the behavior of free-living marine organisms is pivotal for many conservation efforts (REF) and examining how change, such as overfishing and climate variability, affects marine ecosystems ${ }^{[1]}$. For this, many researchers have turned to animal-attached tag technology ${ }^{\text {[2] }}$ where study animals carry archival 'smart' tags that record a suite of parameters to quantify particular aspects of their behavioral ecology or physiology ${ }^{[2]}$. This approach, though powerful, is challenging ${ }^{[3-8]}$, not least because the marine environment is corrosive and tag-carrying animals may expose their devices to extremes in pressure and temperature ${ }^{[9]}$. Almost two decades ago, researchers suggested that magnetic sensing systems could be attractive for monitoring some aspects of animal behavioral ecology. Not only are magnetic fields well tolerated by marine animals ${ }^{[8]}$, but also the magnetic properties exhibited by magnets in water are well defined ${ }^{[10]}$. In particular though, the approach advocated the use of a magnet attached to the study animal on a moving element, such as a limb, that changed position with respect to another animal body part, such as on the animal trunk, on which a magnetic field sensor was mounted. In this case, movement of the limb with respect to the body was apparent via changes in the magnetic field intensity recorded by the sensing system ${ }^{[10]}$. The specifics of the early measuring systems consisted of a magnetic monitoring system composed of a small neodymium magnet coupled with a Hall-effect magnetic field sensor. Indeed, such systems provided critical data in areas as diverse as the feeding behavior ${ }^{[10]}$, limb movements ${ }^{[11]}$, respiration ${ }^{[12]}$, defecation and heart rates ${ }^{[13]}$ of a number of marine vertebrates. Rare earth and ferrite magnets 
have also latterly been utilized in systems exploring spawning of fish ${ }^{[14]}$ and foraging activities of marine homeotherms ${ }^{[15-18]}$.

A little discussed problem in these studies, however, is the effect of the stability of the attachment of the magnet on the quality of the signal. In particular, small magnets (ideal for minimizing the detrimental effects of tags ${ }^{[19]}$ attached to flexible surfaces, such as on animal skin, fur or feathers, can produce large changes in magnetometer response as a result of system mechanical instability. This is particularly the case in tri-axial magnetometers, which are beginning to be used in studies of animal behavior ${ }^{[20]}$, because they are sensitive to both magnet angle and distance between magnet and sensor ${ }^{[20]}$.

In order to provide a more versatile solution for magnetic-based underwater monitoring systems, we propose the use of composite magnets. Mixing magnetic fillers into a soft and flexible carrier matrix has been recently proposed for a new generation of magnetic composite materials ${ }^{[8,21,22]}$ and exploited in biomedical ${ }^{[23-26]}$, robotics ${ }^{[27-31]}$, and automotive ${ }^{[32-34]}$ fields. The formability, multi-directional deflection, low weight and tailored magnetic properties of such flexible composite magnets allow for prescribed magnets to be constructed so that their attachment to animals is mechanically stable. Since they can mirror the body contours and have much less intrusive attachment along the body of animals, the drag force ${ }^{[19]}$ and the likelihood that animals will be disturbed by the physical presence of the system are, thereby, reduced ${ }^{[35]}$. By merging NdFeB magnetic powder with PDMS, we generate a material that can be permanently magnetized, offering both the advantages of hard-magnetic materials, as well as the compliance, flexibility and minimal weight of a polymer. PDMS offers low-cost, rapid and simple fabrication with a high degree of freedom with respect to the shapes, chemical resistance and biocompatibility of the system ${ }^{[36-38]}$. While progress has been made in the area of magnetic nanoparticles ${ }^{[39]}, \mathrm{NdFeB}$ micro powder is still an attractive choice, due to the high energy product, large coercive field and remanent induction ${ }^{[40,41]}$. Isotropic $\mathrm{NdFeB}$ powder is also inexpensive, due to the comparatively large abundance of $\mathrm{Nd}$ and $\mathrm{Fe}$, and has good thermal 
aging characteristics ${ }^{[40-42]}$. As a result of this, NdFeB-based composites have been proposed for applications such as soft MEMs ${ }^{[43,44]}$ microfluidic pressure sensitive valves ${ }^{\text {[45] , }}$ micromanipulation tools ${ }^{[46]}$, microrobots ${ }^{[27]}$ or energy harvesting ${ }^{[65]}$.

For the application of a magnetic composite in marine applications, the susceptibility of the composite to oxidation, biofouling and any corrosive reactions is critically important. In this paper, we report on the development of flexible magnetic NdFeB-PDMS composite magnets with widely tunable properties and study the effects of a seawater environment on the performance of the magnets. We also propose a solution that avoids deterioration of the magnetic properties when the composite is exposed to seawater over extended periods of time, while maintaining the mechanical flexibility. The composite magnets were tested by combining them with magnetic sensors and attaching them to animals. The magnetic animal monitoring system was then used to detect animal movement via a wireless readout system.

\section{Fabrication and Characterization}

\subsection{Fabrication of composite magnets}

The fabrication of the composite magnets comprises four steps; molding, particle alignment, curing and demolding (Figure 1). The fabrication process includes an optional optimization step to obtain a higher remanent magnetization than previously reported ${ }^{[47-49]}$, by particle alignment using a magnetic field, prior to curing.

The preparation of PDMS (Dow Corning Corp. Slygard® 184) includes mixing the elastomer and the curing agent at a 10:1 weight ratio. Composite materials were prepared by dispersing $\mathrm{NdFeB}$ microparticles (Molycorp MQP-16-7FP, $5 \mu \mathrm{m}$ average diameter) into the PDMS, and mixing them thoroughly by mechanical stirring. Concentrations of $10 \%$ wt, $50 \%$ wt and $70 \%$ wt were prepared to study the effects on the mechanical flexibility and magnetic properties. The composites were patterned by pouring them into poly(methyl methacrylate) molds fabricated using a $\mathrm{CO}_{2}$ laser cutter (Universal Laser Systems Inc.PLS 6.75). Air bubbles 
were eliminated by vacuum desiccation. A flat top surface of magnets was obtained by utilizing a casting blade/micrometer adjustable applicator (SH1117/100, Sheen). For the optional alignment of the magnetic fillers along a preferential direction, the composite was subjected to a unidirectional magnetic field of $1.5 \mathrm{~T}$ generated by an electromagnet. Finally, the composite was cured at $90{ }^{\circ} \mathrm{C}$ for one hour.

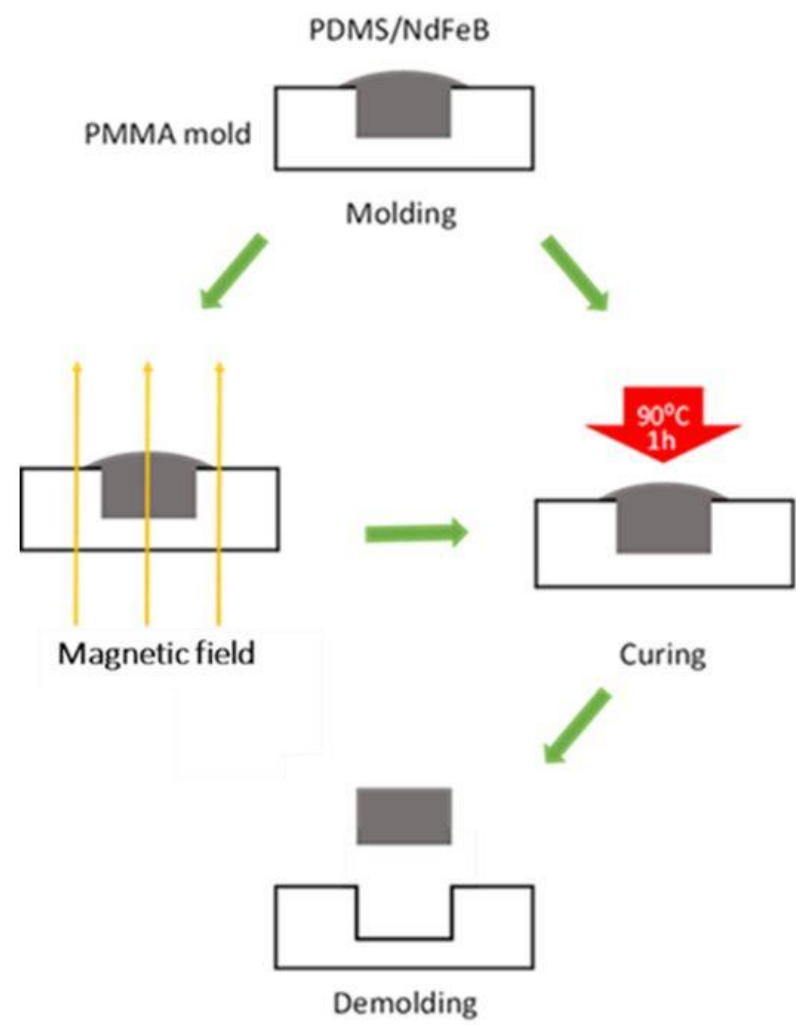

Figure 1. Schematic diagram of the composite magnet fabrication process. The NdFeB-PDMS material mixture is formed inside a PMMA mold. It is then cured and released from the mold. For optimized results, the magnetic particles are aligned prior to curing with a magnetic field.

Although it has been found that varying the $\mathrm{Nd}$ content can decrease the oxidation rate of $\mathrm{NdFeB}$ alloys ${ }^{[49]}$, the goal of this study was to develop a more effective solution that does not compromise the magnetic properties while maintaining the flexibility of the composite magnets. To this end, different protective coatings were tested, including Parylene C, UV resin, epoxy, acrylic and parafilm. The magnetic composites were encapsulated in the following ways: 
1.) The Parylene C (poly(dichloro-p-xylylene)) coating was applied using the PDS 2010 Parylene Deposition System (SCS). With a Parylene granule of $2 \mathrm{mg}$, a coating layer thickness of $1 \mu \mathrm{m}$ was obtained. Further details of this process can be found elsewhere ${ }^{[50]}$.

2.) Clear epoxy adhesive (ALTECO F-05) was applied using disposable polyethylene pipets and then cured for 4 minutes at room temperature.

3.) Flexible photopolymer resin (FLFLGR01, Formlabs) was applied with disposable polyethylene pipets. It was then exposed to ultraviolet light in a CL-1000 UV Crosslinker for 15 minutes to cure.

4.) Acrylic (VCF Films) and 5.) Parafilm "M" (American National Can TM) were applied using outgassing effects created by vacuum soldering.

\subsection{Characterization of composite magnets}

The optical microscopy image in Figure 2a shows the cross section of a composite magnet that was magnetized after curing with $\mathrm{NdFeB}$ concentration of $50 \%$ wt, revealing a relatively homogeneous distribution of the magnetic powder in the polymer matrix. Figure $2 \mathrm{~b}$ shows an image of a composite magnet that was magnetized before curing, revealing that the different processing methods have no effect on the particle distribution, as there is no apparent difference in the particle arrangement.

This was achieved by mixing the powder thoroughly via mechanical stirring and without any additional measure to reduce agglomeration, such as using surfactants ${ }^{[51],[52]}$ or hydroxypropyl cellulose (HPC) $)^{[53]}$.

The composite magnets showed a high mechanical flexibility (Figure 2c), enabling their attachment to marine animals with minimal impact on their behavior. This feature allows the magnets not only to conform to the body curvature of marine animals, but also to follow their body movements. The numbers of species that can be explored and the possible applications 
are also increased, due to the high formability of NdFeB/PDMS composite magnets (Figure 2d).

Compared to a typical commercial permanent magnet (CK\&J Magnetics, NdFeB grade N52, $\left.\mathrm{V}=0.13 \mathrm{~cm}^{3}, 1.27 \mathrm{~g}\right)$, composite magnets of the same volume with $50 \% \mathrm{wt}$ and $10 \% \mathrm{wt}$ were 3 times and 13 times lower in weight, respectively. This is a crucial aspect, since the low weight of the composite magnets poses less burden to marine animals, particularly in species, where overall body density is finely balanced with that of seawater.

a)

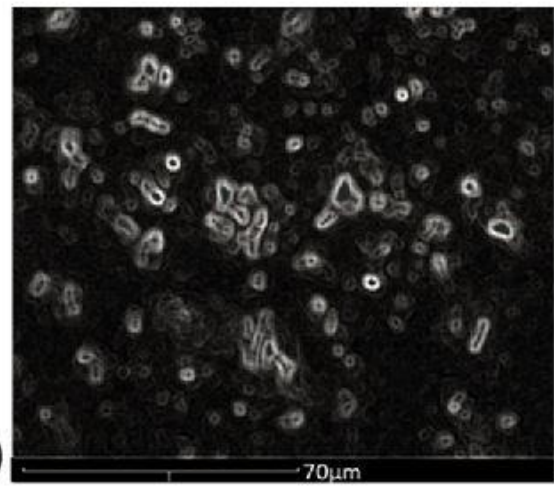

c)

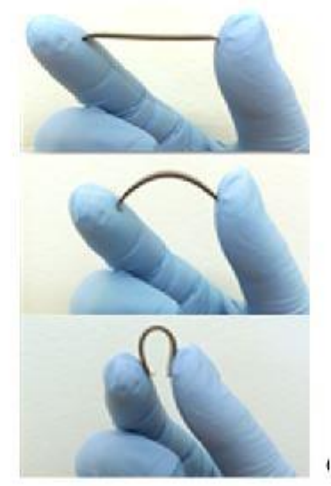

b)

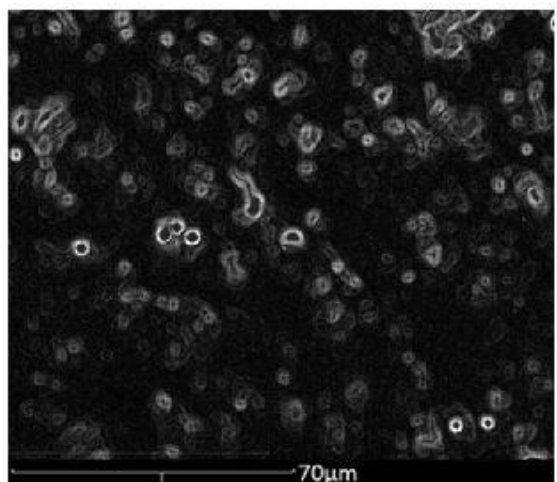

d)

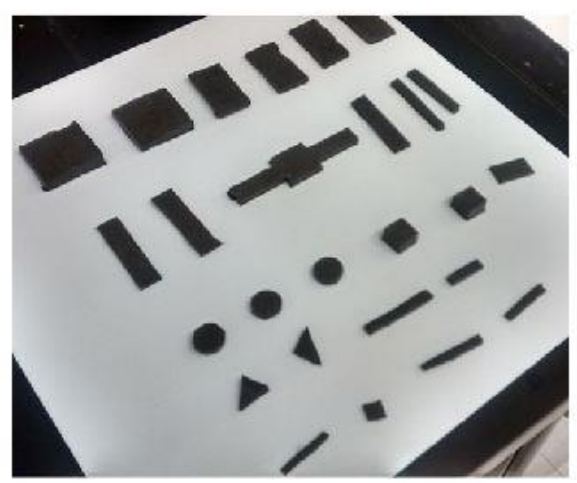

Figure 2 a) Optical microscopy image of the cross-section of a regular magnetic composite sample (magnetized after curing) and b) optimized composite sample (magnetized before curing) c) Flexible NdFeB-PDMS magnetic composite ribbon bent by hand. d) Examples of different shapes and sizes of composite magnets.

The magnetic properties were characterized for cylindrical composite magnets with $1 \mathrm{~cm}$ in length and $0.2 \mathrm{~cm}$ in diameter at room temperature using a vibrating sample magnetometer. Composite magnets with $\mathrm{NdFeB}$ concentrations of $10 \% \mathrm{wt}, 50 \% \mathrm{wt}$ and $70 \% \mathrm{wt}$ were investigated, and samples with $50 \%$ wt were studied with and without $\mathrm{NdFeB}$ particle 
alignment. The magnetization curves revealed that the composite magnets have the same coercivity $\left(\mathrm{H}_{\mathrm{c}}\right)$ of $(5.4 \pm 0.1) \mathrm{kOe}$, independent of the concentration (Figure 3), while the remanent and saturation magnetizations had a positive linear relationship with the concentration (Figure 4a). According to the manufacturer's specification, the value of $\mathrm{H}_{\mathrm{i}}$ of $\mathrm{NdFeB}$ particles is $(4.7-5.5) \mathrm{kOe}{ }^{[54]}$, which indicates that the magnetic properties of the particles were not affected by the fabrication process. It also shows that the magnetostatic interaction between the particles at these concentrations did not lead to a considerable agglomeration.

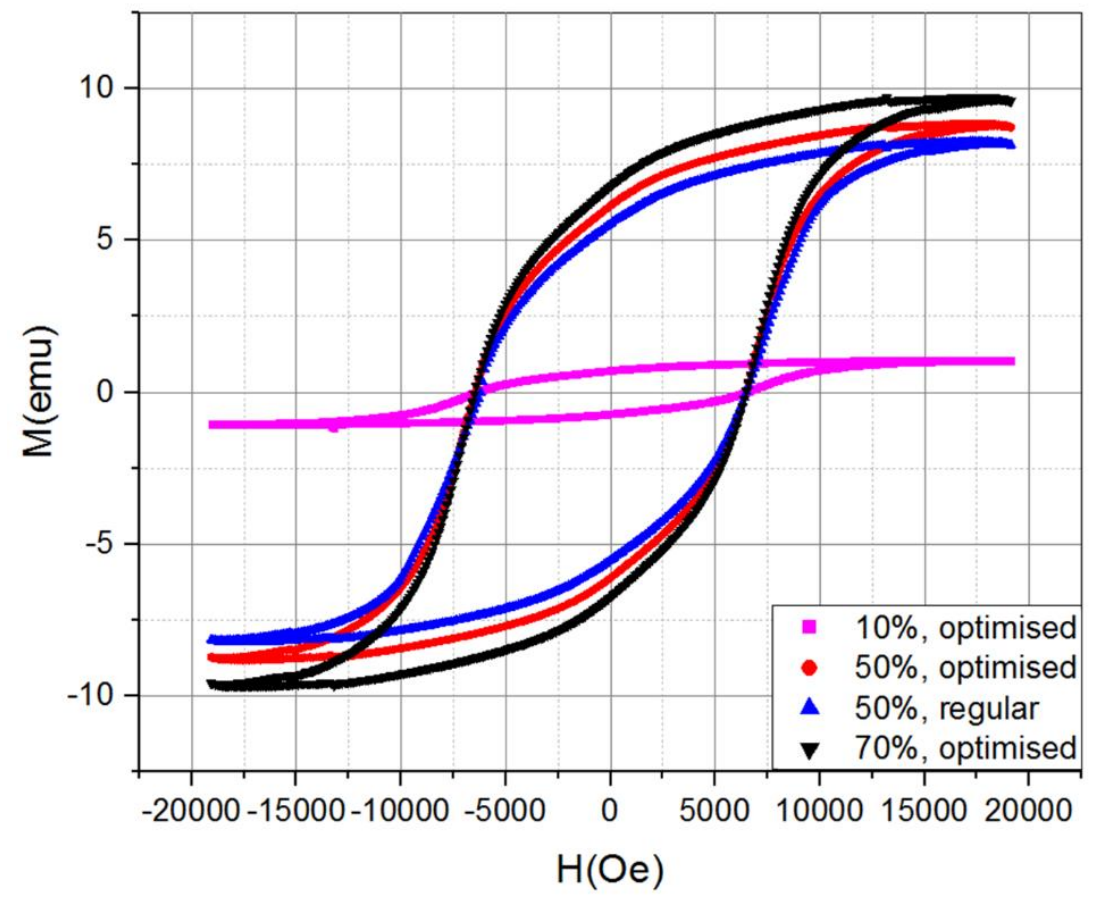

Figure 3. Magnetization curves of magnetic composite magnets with a cylindrical shape. The percentage values indicate the NdFeB powder concentration. The NdFeB powder particles were either aligned (optimized) or not aligned (regular) before curing.

The optimization step, in which a magnetic field is applied prior curing, improved the values of saturation and remanence magnetizations of the composite magnets (Figure 3 red and blue loops, respectively). The remanent magnetization, which is the most relevant parameter for the intended application, increased by $16 \%$, due to the alignment of the particles' anisotropy axis with the applied magnetic field. In addition, the magnetic properties were reproducible for 
different samples from the same magnetic composite batch, confirming the homogenous dispersion of the NdFeB powder inside of the PDMS.
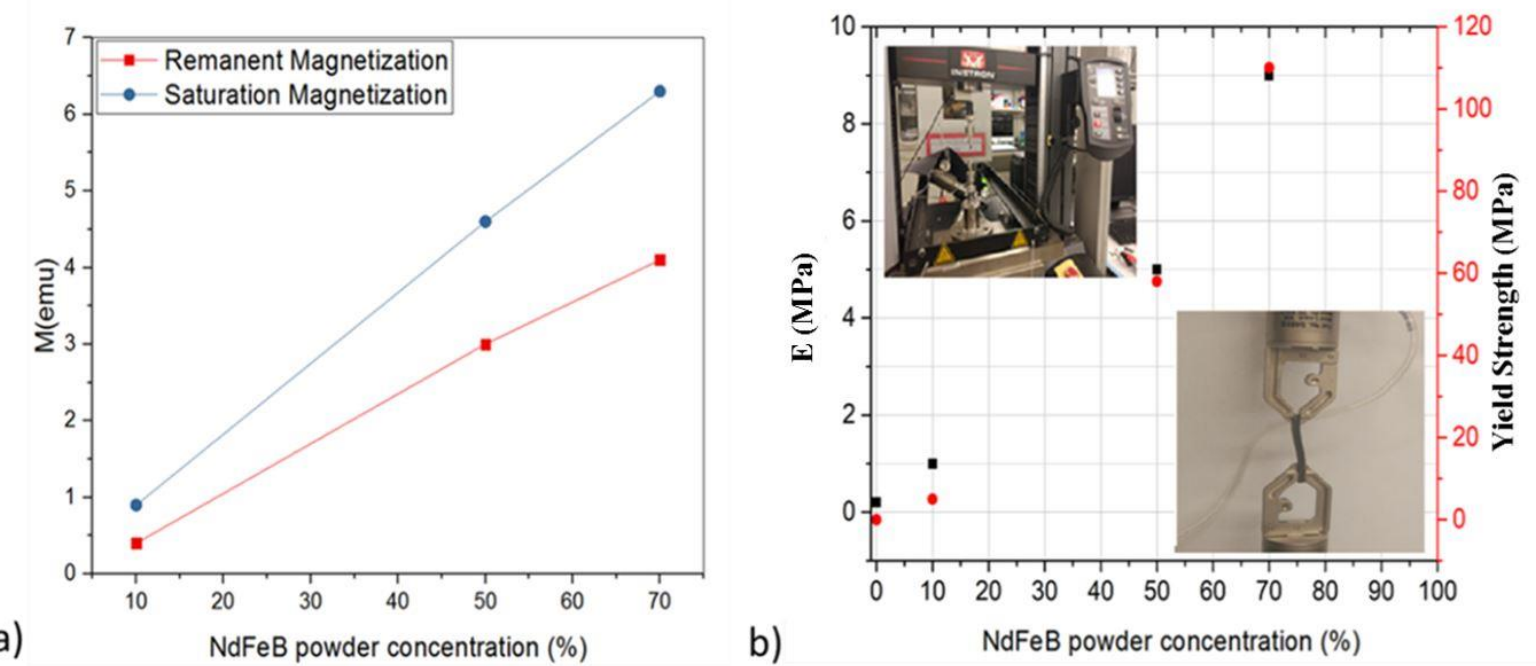

Figure 4 a) Remanent and saturation magnetizations of composite magnets as a function of the $N d F e B$ powder concentration; b) Young's modulus, E, and Yield strength of composite magnets as a function of $\mathrm{NdFeB}$ powder concentration.

In order to study the mechanical properties of the composite magnets, a tensile test was performed using an electromechanical pull tester (Instron 5900-Series). Figure $4 \mathbf{b}$ shows the Young's modulus and Yield strengths of magnetic composite samples with different $\mathrm{NdFeB}$ concentrations taken from the stress/strain diagrams. The Young's modulus increased with increasing amounts of $\mathrm{NdFeB}$ powder, due to the reduced volume of the polymer bearing the stress in the powder network. A similar result has been reported for NdFeB/epoxy composites [55, 56]. The Young's modulus of $\mathrm{NdFeB}$ magnets is $150 \mathrm{GPa}{ }^{[57]}$, which is nearly $10^{5}$ higher than the one of the composite magnets. A similar trend can be observed for the yield strength, which increases with the filler content.

\section{Results and Discussion}

\subsection{Magnetic properties of composite magnets in sea water}


$\mathrm{NdFeB}$ powder is known for its poor resistance to corrosion, ostensibly limiting its use in underwater applications ${ }^{[58,59]}$. In sea water, a deterioration of the magnetic properties occurs, due to the oxidizing environment, following an inverse parabolic rate law. Biofouling is another process that creates adverse effects on immersed magnetic materials, further limiting their utilization and accelerating the corrosion ${ }^{[60]}$.

Therefore, the magnetic properties of NdFeB-PDMS composite magnets deployed in sea water were investigated for different lengths of time. To this end, cylindrical composite magnets were made with $50 \%$ wt of magnetic powder and deployed at the Al Fahal reef in the Cental Red Sea (geographic coordinates: $22.25285^{\circ} \mathrm{N}, 38.96123^{\circ} \mathrm{E}$, average salinity: $\sim 35 \%$, average temperture $\sim 22^{\circ} \mathrm{C}$ ). The results showed that both the remanence and the saturation magnetization followed the same trend, deteriorating quickly (Figure 5), with the greatest changes happening during the first 3 days. This is the case for both composite magnets made with optimized and regular fabrication processes. During the first 3 days, between $30 \%$ and $38 \%$ of the magnetization was lost and this figure increased to $60 \%$ after 51 days.

Biofouling on the composite magnets was evaluated using both accumulation of matter in terms of the surface area covered and weight of the biofoulants. Using ImageJ software ${ }^{[61,62]}$, the area of the composite magnets surface covered by biofoulants after 51 days was found to be $13.2 \%$ (inset in Figure 5). The biomass of the biofoulants was $10.2 \mathrm{mg} / \mathrm{cm}^{2}$, as determined by gently scrapping the surface of the magnets until no fouling was visible and weighing the fouling materials removed. This measurement represents the upper limit of what would be expected given that biofouling on sedentary substrates is likely greater than when the material is attached to mobile organisms. In addition, a visual assessment found that other materials, such as PMMA and metals (deployed at the same time as composite magnets) had more biofouling. Given the rapid decline in remanent magnetization, which occurred much faster than visible biofouling, corrosion is likely the primary factor limiting the longevity of the magnets. 


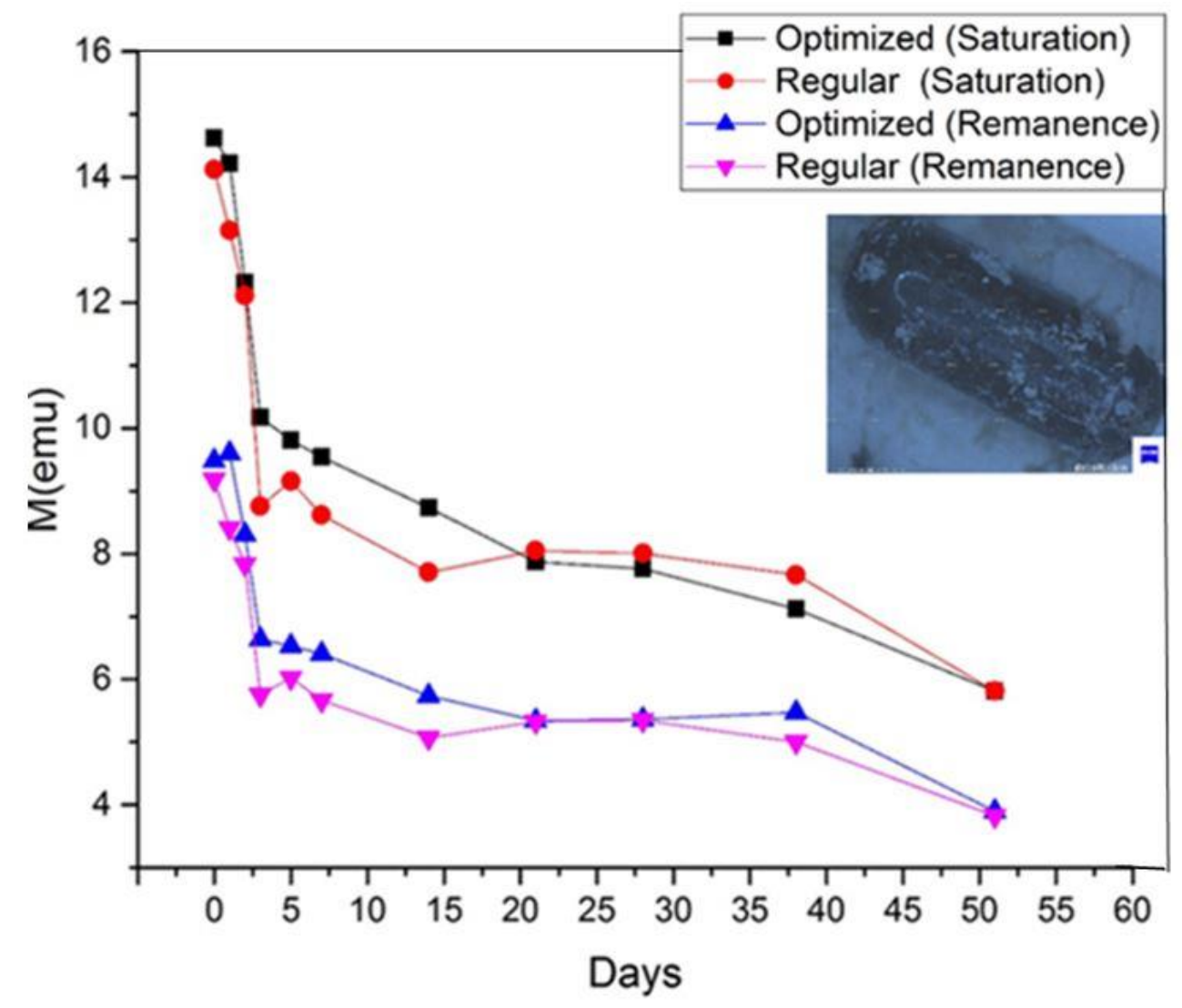

Figure 5. Saturation and remanent magnetizations of cylindrical composite magnets with $50 \%$ wt NdFeB powder in sea water. The NdFeB powder particles were either aligned (optimized) or not aligned (regular) before curing. The inset shows a stereoscope image of a composite magnet after 51 days in sea water, revealing biofouling on its surface.

\subsection{Polymer coatings for corrosion protection}

The capability of the polymer coatings to preserve the composite magnet properties was evaluated over a period of 21 days. Coatings slow down the corrosion compared to the unprotected composite magnets (Figure 6). However, all polymers had limited success in protecting the magnetic properties, except for Parylene $\mathrm{C}$, in which case the remanent magnetization od composite magnet remained stable over 21 days. 


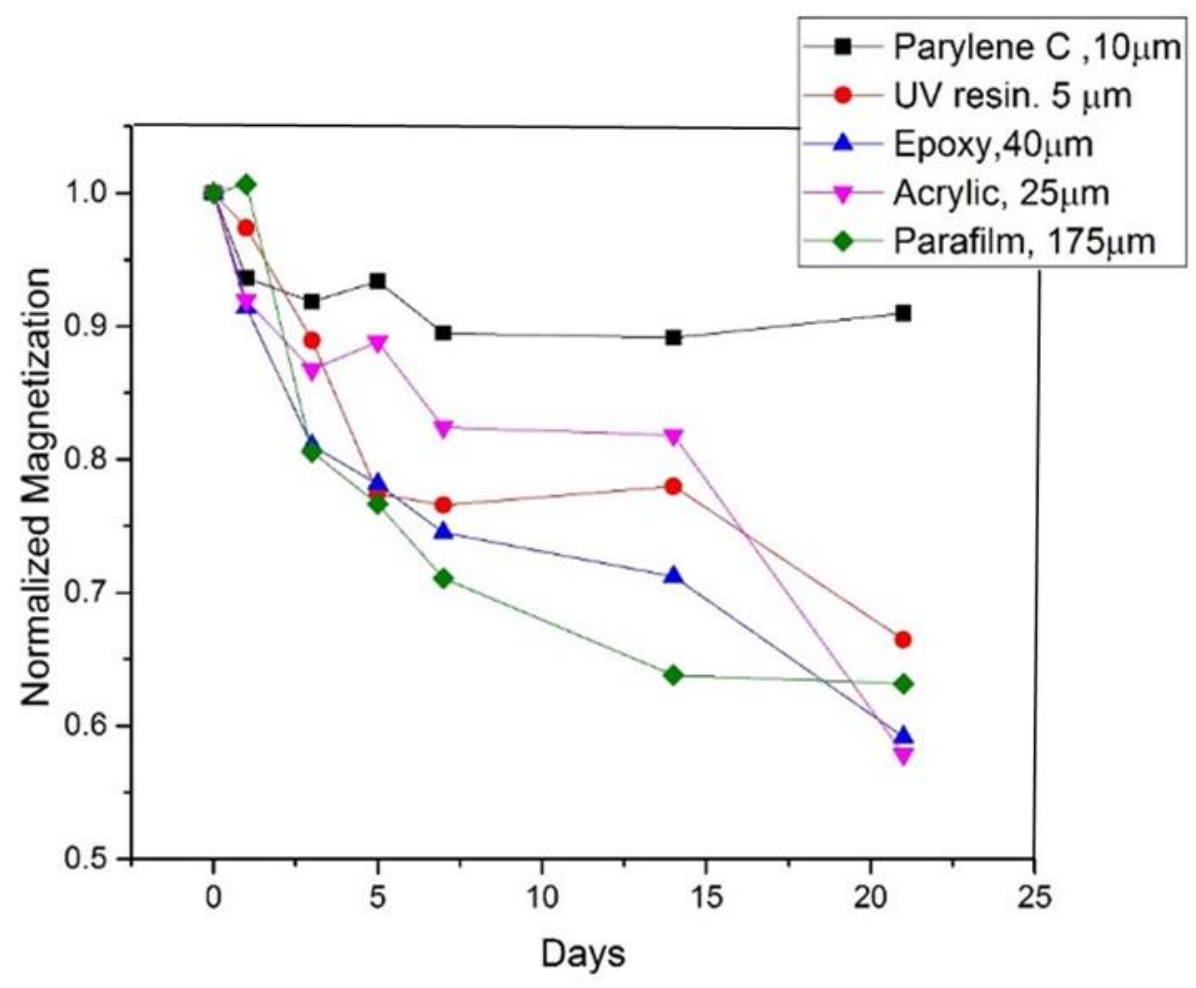

Figure 6. Remanent magnetization of composite magnets coated with different polymers and deployed in the Red Sea.

Based on this, Parylene $\mathrm{C}$ was used in further investigations and samples of magnetic composites were coated with thicknesses of $2 \mu \mathrm{m}$ and $4 \mu \mathrm{m}$ thickness. The parylene $\mathrm{C}$ coatings preserved magnetization for nearly 65 days for $2 \mu \mathrm{m}$ and 82 days for $4 \mu \mathrm{m}$ coatings (Figure 7). This may be explained by the low water and gas permeability of Parylene $C^{[39,63]}$. Parylene $C$ also exhibits a high biocompatibility, is optically transparent and flexible ( 4 GPa of Young's modulus) ${ }^{[64-67]}$, which are additional features making it suitable for marine animal monitoring applications. 


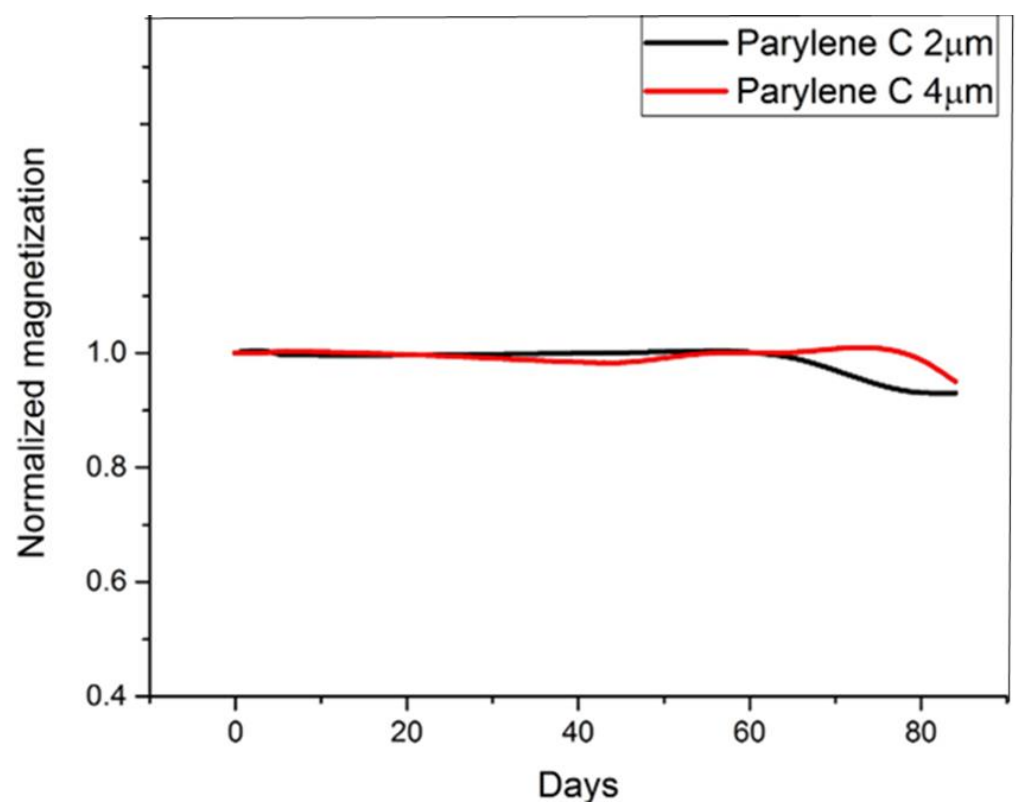

Figure 7. Remanence magnetization of magnetic composites coated with $2 \mu \mathrm{m}$ and $4 \mu \mathrm{m}$ of Parylene $C$ and deployed in the Red Sea.

\subsection{Marine animals monitoring}

Much basic knowledge about the behavior of free-living marine organisms can be obtained by examining animal movement, both translocational and otherwise. The versatility of the composite magnets should catalyze the development of more compelling and flexible underwater magnetic monitoring systems based on magnetic field sensors in combination with the developed corrosion-resistant and bendable composite magnets. By way of demonstration, our application had the stray field of the composite magnet measured by a magnetic tunnel junction sensor (TMR2305, Multi Dimension, Inc), finding the resulting signal (read via an inhouse wireless communication module ${ }^{[68,69]}$ ) was a function of the distance between the sensor and the composite magnet. With regard to location, the composite magnets were attached to animals using waterproof velcro (VLC02, Velcro® Brand Marine Grade Hook and Loop) and pure epoxy compound (Subcoat S, Veneziani Yachting). In case of a giant clam, (Tridacna gigas), an important component of coral reefs ecosystems in the Red Sea, the sensor was mounted on one valve margin and the magnet on the opposite valve margin, which provides multiple response variables related to mantle opening (Figure 8a). In case of crabs (Libinia emarginata), the sensor was mounted on the carapace and the magnet on the third leg (merus 
section), in order to monitor the physical activity level of the crab (Figure 8b). Finally, in case of tortoises (Aldabrachelys gigantea), the sensor was mounted on the carapace (marginal scutes), and the magnet on the front right leg in order to monitor the animal's physical activity (Figure 8c). The magnetic monitoring system operated reliably over distances of up to $10 \mathrm{~cm}$ between the sensor and the composite magnet, which was the maximum required for those three animals. No sign of deterioration of the magnets was found during the experiments, which lasted up to 24 hours. . The signal derived from the animal monitoring experiments enables extraction pivotal information such as levels of activity over time (e.g. day time versus night) or as a function of proximity to predators etc.
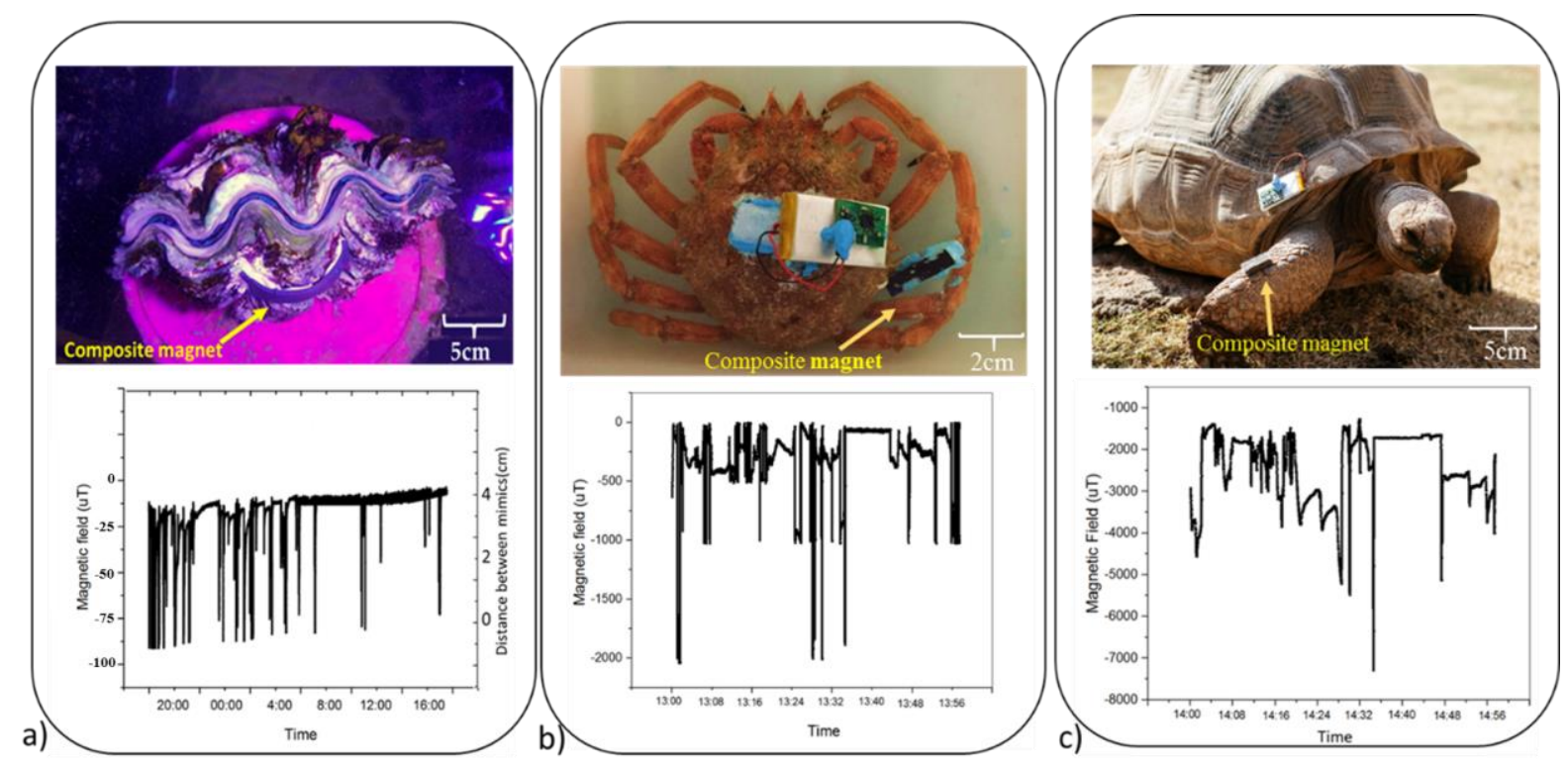

Figure 8. Marine and terrestrial animal monitoring experiments utilizing composite magnets and commercially available magnetic sensors. a) giant clam, b) crab and c) giant tortoise. The bottom images show the sensor signal over time.

\section{Conclusion}

NdFeB-PDMS composite magnets were studied for underwater marine monitoring applications. The fabrication process involves minimal complexity and is highly versatile with respect to the shape of the magnets. The remanent magnetization can be enhanced by $16 \%$ with an additional step, during which the magnetic particles are aligned by a magnetic field. The influence of the filler concentration on magnetic and mechanical properties was determined and 
showed that they can be tailored within a wide range. The composite magnets have a large bending flexibility with a Young's Modulus of $\sim 10^{5}$ times lower than the one of a bulk permanent magnet, in case of $10 \%$ wt particle concentration. This means that they can readily conform to the curved surface of marine animals, as demonstrated by mounting composite magnets to a giant clam, crabs and turtles. The weight of the composite magnets can also be considerably reduced, by a factor of 13 in the case of $10 \%$ wt particle concentration, compared to a bulk magnet. The proposed fabrication is cost-effective, due to the rapid production of reusable polymer molds by a $\mathrm{CO}_{2}$-laser system. The performance of the flexible magnetic composites was tested in sea water, where they quickly showed a reduction in the remanent magnetization, due to corrosion and biofouling. A coating study revealed that Paralyne $\mathrm{C}$ is the most suitable and efficient polymer for protecting the composite from deterioration, providing corrosion resistance, flexibility and biocompatibility. The magnetic properties of the composite magnets were maintained for nearly three months while being deployed in the particularly harsh environment, with high salinity, temperature and growth rates of fouling communities, of the Red Sea. The composite magnets were tested in combination with magnetic sensors on three different animals underwater and on land using a reliable wireless monitoring system. The results show the suitability of the composite magnets for marine applications. Their low weight, mechanical flexibility and deformability provide the features necessary to tackle new applications and study species with a wide range in body size and morphology, which is not possible with rigid permeant magnets.

\section{Acknowledgment}

This research is a contribution to the CAASE project funded by King Abdullah University of Science and Technology (KAUST) under the KAUST Sensor Initiative. We thank the staff and leadership of the Oceanografic in Valencia for their help and support during the sensor tests with turtles and crabs 
Received: ((will be filled in by the editorial staff))

Revised: ((will be filled in by the editorial staff)) Published online: ((will be filled in by the editorial staff))

[1] Maxwell, Sara M., Elliott L. Hazen, Steven J. Bograd, Benjamin S. Halpern, Greg A. Breed, Barry Nickel, Nicole M. Teutschel "Cumulative human impacts on marine predators." Nature communications 4 (2013): 2688.

[2] Ropert-Coudert, Yan, and Rory P. Wilson. "Trends and perspectives in animal-attached remote sensing." Frontiers in Ecology and the Environment 3.8 (2005): 437-444.

[3] Cooke, Steven J., Scott G. Hinch, Martin Wikelski, Russel D. Andrews, Louise J. Kuchel, Thomas G. Wolcott, and Patrick J. Butler. "Biotelemetry: a mechanistic approach to ecology." Trends in ecology \& evolution 19, no. 6 (2004): 334-343.

[4] Block, Barbara A., Daniel P. Costa, George W. Boehlert, and Randy E. Kochevar. "Revealing pelagic habitat use: the tagging of Pacific pelagics program." Oceanologica Acta 25, no. 5 (2002): 255-266.

[5] Lander, Michelle E., Todd Lindstrom, Matthew Rutishauser, Albert Franzheim, and Melinda Holland. "Development and field testing a satellite-linked fluorometer for marine vertebrates." Animal Biotelemetry 3, no. 1 (2015): 40.

[6] Franklin, Paul, and C. I. N. D. Y. Baker. "Fish passage research in the Southern Hemisphere: Challenges, lessons and the need for novel solutions." International Symposium on Ecohydraulics, Melbourne, Australia. 2016.

[7] Donaldson, M. R., G. D. Raby, V. N. Nguyen, S. G. Hinch, D. A. Patterson, A. P. Farrell, M. A. Rudd et al. "Evaluation of a simple technique for recovering fish from capture stress: integrating physiology, biotelemetry, and social science to solve a conservation problem." Canadian Journal of Fisheries and Aquatic Sciences 70, no. 1 (2013): 90-100. 
[8] Rigg, Damian P., Stirling C. Peverell, Mark Hearndon, and Jamie E. Seymour. "Do elasmobranch reactions to magnetic fields in water show promise for bycatch mitigation?." Marine and Freshwater Research 60, no. 9 (2009): 942-948

[9] Guinet, Christophe, Jade Vacquié-Garcia, Baptiste Picard, Guillaume Bessigneul, Yves Lebras, Anne Cécile Dragon, Morgane Viviant, John PY Arnould, and Frédéric Bailleul. "Southern elephant seal foraging success in relation to temperature and light conditions: insight into prey distribution." Marine Ecology Progress Series 499 (2014): 285-301.

[10] Wilson, R., Antje Steinfurth, Y. Ropert-Coudert, A. Kato, and M. Kurita. "Lip-reading in remote subjects: an attempt to quantify and separate ingestion, breathing and vocalisation in free-living animals using penguins as a model." Marine Biology 140, no. 1 (2002): 17-27.

[11] Wilson, R., and Nikolai Liebsch. "Up-beat motion in swinging limbs: new insights into assessing movement in free-living aquatic vertebrates." Marine Biology 142.3 (2003): 537-547. [12] Wilson, Rory P., Alejandro Simeone, Guillermo Luna-Jorquera, Antje Steinfurth, Sue Jackson, and Andreas Fahlman. "Patterns of respiration in diving penguins: is the last gasp an inspired tactic?." Journal of Experimental Biology 206, no. 10 (2003): 1751-1763

[13] Wilson, Rory P., Alejandro Scolaro, Flavio Quintana, Ursula Siebert, M. Thor Straten, K. Mills, Ilka Zimmer et al. "To the bottom of the heart: cloacal movement as an index of cardiac frequency, respiration and digestive evacuation in penguins." Marine Biology 144, no. 4 (2004): 813-827.

[14] Metcalfe, J. D., M. C. Fulcher, S. R. Clarke, M. J. Challiss, and S. Hetherington. "An archival tag for monitoring key behaviours (feeding and spawning) in fish." In Tagging and Tracking of Marine Animals with Electronic Devices, pp. 243-254. Springer, Dordrecht, 2009.

[15] Ropert-Coudert, Y., A. Kato, Nikolai Liebsch, Rory P. Wilson, G. Muller, and E. Baubet. "Monitoring jaw movements: a cue to feeding activity." Game and Wildlife Science 21, no. 1 (2004): 1-20. 
[16] Liebsch, Nikolai. Measurement offeeding and activity in air-breathing marine vertebrates using the Hall effect. Diss. Diploma Thesis, Christian-Albrechts-University Kiel, Germany, 2002.

[17] Hanuise, Nicolas, Charles-André Bost, William Huin, Arnaud Auber, Lewis G. Halsey, and Yves Handrich. "Measuring foraging activity in a deep-diving bird: comparing wiggles, oesophageal temperatures and beak-opening angles as proxies of feeding." Journal of Experimental Biology 213, no. 22 (2010): 3874-3880.

[18] Hazen, Elliott L., Sara M. Maxwell, Helen Bailey, Steven J. Bograd, Mark Hamann, Philippe Gaspar, Brendan J. Godley, and George L. Shillinger. "Ontogeny in marine tagging and tracking science: technologies and data gaps." Marine Ecology Progress Series 457 (2012): 221-240.

[19] Vandenabeele, S. P., E. L. C. Shepard, D. Grémillet, P. J. Butler, G. R. Martin, and R. P. Wilson. "Are bio-telemetric devices a drag? Effects of external tags on the diving behaviour of great cormorants." Marine Ecology Progress Series 519 (2015): 239-249.

[20] Shepard, Emily LC, Rory P. Wilson, Flavio Quintana, Agustina Gómez Laich, Nikolai Liebsch, Diego A. Albareda, Lewis G. Halsey et al. "Identification of animal movement patterns using tri-axial accelerometry." Endangered Species Research 10 (2008): 47-60. [21] Marchi, Sophie, Alberto Casu, Franco Bertora, Athanassia Athanassiou, and Despina Fragouli. "Highly magneto-responsive elastomeric films created by a two-step fabrication process." ACS applied materials \& interfaces 7, no. 34 (2015): 19112-19118.

[22] Wang, Weisong, Zhongmei Yao, Jackie C. Chen, and Ji Fang. "Composite elastic magnet films with hard magnetic feature." Journal of Micromechanics and Microengineering 14, no. 10 (2004): 1321. 
[23] Y. Yi, A. Zaher, O. Yassine, J. Kosel, I.G. Foulds, “A remotely operated drug delivery system with an electrolytic pump and a thermoresponsive valve," Biomicrofluidics, 9, 052608 (2015); doi: 10.1063/1.4927436

[24] A. Zaher, S. Li, O. Yassine, N. Khashab, N. Pirmoradi, L. Lin, J. Kosel: “Osmotically driven drug delivery through remote-controlled magnetic nanocomposite membranes". Biomicrofluidics, 9, 5 (2015). DOI: 10.1063/1.4931954

[25] O. Yassine, A. Zaher, E. Li, E.A. Qiang, Alfadhel, J.E. Perez, M. Kavaldzhiev, M.F. Contreras, S. Li , S. T. Thoroddsen, N. M. Khashab, and J. Kosel, "Highly Efficient Thermoresponsive Nanocomposite for Controlled Release Applications,” $\quad$ Scientific Reports 6, 28539, (2016). doi:10.1038/srep28539.

[26] Yu. P. Ivanov, A. Alfadhel, M. Alnassar, J. E. Perez, M. Vazquez, A. Chuvilin and J. Kosel, "Tunable magnetic nanowires for biomedical and harsh environment applications," Scientific Reports, 6, 24189 (2016). doi:10.1038/srep24189

[27] Iacovacci, V., G. Lucarini, C. Innocenti, N. Comisso, P. Dario, L. Ricotti, and A. Menciassi. "Polydimethylsiloxane films doped with NdFeB powder: magnetic characterization and potential applications in biomedical engineering and microrobotics." Biomedical microdevices 17, no. 6 (2015): 112.

[28] Alfadhel, J. Kosel, "Magnetic Nanocomposite Cilia Tactile Sensor,” Advanced Materials, 27, 47, pp. 7888-7892 (2015). DOI: 10. 1002/adma. 201504015

[29] Alfadhel, Ahmed, Mohammed Asadullah Khan, Susana Cardoso de Freitas, and Jürgen Kosel. "Magnetic tactile sensor for braille reading." IEEE Sensors Journal 16, no. 24 (2016): 8700-8705.

[30] P Ribeiro, Pedro, Mohammed Asadullah Khan, Ahmed Alfadhel, Jürgen Kosel, Fernando Franco, Susana Cardoso, Alexandre Bernardino, Alexander Schmitz, José Santos-Victor, and 
Lorenzo Jamone. "Bioinspired ciliary force sensor for robotic platforms." IEEE Robotics and Automation Letters 2, no. 2 (2017): 971-976.

[31] Alfadhel, Ahmed, Mohammed Asadullah Khan, Susana Cardoso, Diana Leitao, and Jürgen Kosel. "A magnetoresistive tactile sensor for harsh environment applications." Sensors 16, no.

[32] Wang, Ru-Min, Shui-Rong Zheng, and Yujun George Zheng. Polymer matrix composites and technology. Elsevier, 2011.

[33] M. Alnassar, Yu. P. Ivanov, J. Kosel, "Flexible Magnetoelectric Nanocomposites with Tunable Properties," Advanced Electronic Materials, 1600081 (2016). Doi: 10.1002/aelm.201600081

[34] Spencer, Kevin, et al. "Established and emerging materials for use as high-field magnet conductors." Advanced Engineering Materials 6.5 (2004): 290-297.

[35] Wilson, Rory P. "Animal behaviour: the price tag." Nature469.7329 (2011): 164-165.

[36] Hemmilä, Samu, Juan V. Cauich-Rodríguez, Joose Kreutzer, and Pasi Kallio. "Rapid, simple, and cost-effective treatments to achieve long-term hydrophilic PDMS surfaces." Applied Surface Science 258, no. 24 (2012): 9864-9875.

[37] Jothimuthu, Preetha, Andrew Carroll, Ali Asgar S. Bhagat, Gui Lin, James E. Mark, and Ian Papautsky. "Photodefinable PDMS thin films for microfabrication applications." Journal of Micromechanics and Microengineering 19, no. 4 (2009): 045024.

[38] Kroner, Elmar, Roya Maboudian, and Eduard Arzt. "Adhesion Characteristics of PDMS Surfaces During Repeated Pull-Off Force Measurements." Advanced Engineering Materials 12.5 (2010): 398-404.

[39] Suber, L., G. Marchegiani, E. S. Olivetti, F. Celegato, M. Coïsson, P. Tiberto, P. Allia et al. "Pure magnetic hard fct FePt nanoparticles: Chemical synthesis, structural and magnetic properties correlations." Materials Chemistry and Physics 144, no. 1-2 (2014): 186-193. 
[40] Ślusarek, B., and I. Dudzikowski. "Application of permanent magnets made from NdFeB powder and from mixtures of powders in DC motors." Journal of Magnetism and Magnetic Materials 239, no. 1-3 (2002): 597-599.

[41] Ma, B. M., J. W. Herchenroeder, B. Smith, M. Suda, D. N. Brown, and Z. Chen. "Recent development in bonded NdFeB magnets." Journal of magnetism and magnetic materials 239, no. $1-3$ (2002): $418-423$.

[42] Rahbar, M., H. Y. Tseng, and B. L. Gray. "High-aspect ratio magnetic nanocomposite polymer cilium." SPIE MOEMS-MEMS. International Society for Optics and Photonics, 2014. [43] Khosla, Ajit. Micropatternable multifunctional nanocomposite polymers for flexible soft MEMS applications. Diss. Applied Science: School of Engineering Science, 2011.

[44] Khosla A, Gray BL (2012) (Invited) Micropatternable multifunctional nanocomposite polymers for flexible soft NEMS and MEMS applications. ECS Trans 45(3):477-494

[45] Hilber, W., and B. Jakoby. "Controlled liquid flow in a microfluidic network with pressure sensitive valves based on polydimethylsiloxane (PDMS)/neodymium (NdFeB) composites." Procedia Engineering 47 (2012): 382-385,.

[46] Diller, Eric, and Metin Sitti. "Three-Dimensional Programmable Assembly by Untethered Magnetic Robotic Micro-Grippers." Advanced Functional Materials 24, no. 28 (2014): 4397 4404.

[47] Drotlef, Dirk-Michael, Peter Blümler, and Aránzazu del Campo. "Magnetically actuated patterns for bioinspired reversible adhesion (Dry and Wet)." Advanced Materials 26.5 (2014): 775-779.

[48] Muljadi, Priyo Sardjono, and Suprapedi. "Preparation and characterization of polymeric composite permanent magnet Nd2Fe14B." AIP Conference Proceedings. Vol. 1719. No. 1. AIP Publishing, 2016.

[49] Chiolerio, Alessandro, Simone Musso, Marco Sangermano, Mauro Giorcelli, Stefano Bianco, Marco Coisson, Aldo Priola, P. Allia, and Alberto Tagliaferro. "Preparation of 
polymer-based composite with magnetic anisotropy by oriented carbon nanotube dispersion." Diamond and Related Materials 17, no. 7-10 (2008): 1590-1595.

[50] Bender, Florian, Kerstin Länge, Nicole Barié, Jun Kondoh, and Michael Rapp. "On-line monitoring of polymer deposition for tailoring the waveguide characteristics of love-wave biosensors." Langmuir 20, no. 6 (2004): 2315-2319.

[51] Inaba, Hideo, Takaaki Inada, Akihiko Horibe, Hiroshi Suzuki, and Hiromoto Usui. "Preventing agglomeration and growth of ice particles in water with suitable additives." International Journal of Refrigeration 28, no. 1 (2005): 20-26.

[52] Rabinow, Barrett E. "Nanosuspensions in drug delivery." Nature Reviews Drug Discovery 3.9 (2004): 785.

[53] Jean, J. H., and T. A. Ring. "Effect of a sterically stabilizing surfactant on the nucleation, growth and agglomeration of monosized ceramic powders." Colloids and Surfaces 29.3 (1988): 273-29

[54] Magnequench Technology Center, http://www.mqitechnology.com

[55] Grujić, A., N. Talijan, D. Stojanović, J. Stajić-Trošić, Z. Burzić, Lj Balanović, and R. Aleksić. "Mechanical and magnetic properties of composite materials with polymer matrix." Journal of Mining and Metallurgy B: Metallurgy 46, no. 1 (2010): 25-32.

[56] Johnston, I. D., D. K. McCluskey, C. K. L. Tan, and M. C. Tracey. "Mechanical characterization of bulk Sylgard 184 for microfluidics and microengineering." Journal of Micromechanics and Microengineering 24, no. 3 (2014): 035017.

[57] Rabinovich, Yu M., V. V. Sergeev, A. D. Maystrenko, V. Kulakovsky, S. Szymura, and H. Bala. "Physical and mechanical properties of sintered $\mathrm{Nd} \cdot \mathrm{Fe} \cdot \mathrm{B}$ type permanent magnets." Intermetallics 4, no. 8 (1996): 641-645. 
[58] Cygan, D. F., and M. J. McNallan. "Corrosion of NdFeB permanent magnets in humid environments at temperatures up to $150^{\circ}$ C." Journal of Magnetism and Magnetic Materials 139.1-2 (1995): 131-138.

[59] Mao, Shoudong, Hengxiu Yang, Zhenlun Song, Jinlong Li, Huagen Ying, and Kefei Sun. "Corrosion behaviour of sintered $\mathrm{NdFeB}$ deposited with an aluminium coating." Corrosion Science 53, no. 5 (2011): 1887-1894.

[60] Chapman, James, and Fiona Regan. "Nanofunctionalized superhydrophobic antifouling coatings for environmental sensor applications-advancing deployment with answers from nature." Advanced Engineering Materials 14.4 (2012).

[61] Abràmoff, Michael D., Paulo J. Magalhães, and Sunanda J. Ram. "Image processing with ImageJ." Biophotonics international 11.7 (2004): 36-42.

[62] Kolesky, David B., Ryan L. Truby, A. Sydney Gladman, Travis A. Busbee, Kimberly A. Homan, and Jennifer A. Lewis. "3D bioprinting of vascularized, heterogeneous cell-laden tissue constructs." Advanced materials 26, no. 19 (2014): 3124-3130.

[63] Li, Wen, Damien C. Rodger, Anderson Pinto, Ellis Meng, James D. Weiland, Mark S. Humayun, and Yu-Chong Tai. "Parylene-based integrated wireless single-channel neurostimulator." Sensors and Actuators A: Physical 166, no. 2 (2011): 193-200.

[64] Liger, Matthieu, Damien C. Rodger, and Yu-Chong Tai. "Robust parylene-to-silicon mechanical anchoring." Micro Electro Mechanical Systems, 2003. MEMS-03 Kyoto. IEEE The Sixteenth Annual International Conference on. IEEE, 2003.

[65] Khan, Mohammed Asadullah, Ahmed Alfadhel, and Jürgen Kosel. "Magnetic nanocomposite cilia energy harvester." IEEE Transactions on Magnetics 52, no. 7 (2016): 1-4.

[66] Chang, Tracy Y., Vikramaditya G. Yadav, Sarah De Leo, Agustin Mohedas, Bimal Rajalingam, Chia-Ling Chen, Selvapraba Selvarasah, Mehmet R. Dokmeci, and Ali 
Khademhosseini. "Cell and protein compatibility of parylene-C surfaces." Langmuir 23, no. 23 (2007): 11718-11725.

[67] Yao, Tze-Jung, Xing Yang, and Yu-Chong Tai. "BrF3 dry release technology for large freestanding Parylene MEMS." Transducers’ 01 Eurosensors $X V$. Springer Berlin Heidelberg, 2001. $652-655$.

[68] Yu, Bin, Lisheng $\mathrm{Xu}$, and Yongxu Li. "Bluetooth low energy (BLE) based mobile electrocardiogram monitoring system." Information and Automation (ICIA), 2012 International Conference on. IEEE, 2012

[69] Kim, Jeonghyun, et al. "Miniaturized Flexible Electronic Systems with Wireless Power and Near-Field Communication Capabilities." Advanced Functional Materials 25.30 (2015): $4761-4767$ 